\title{
Arm Locking for the Laser Interferometer Space Antenna
}

\author{
P. G. Maghami, J. I. Thorpe, and J. Livas \\ NASA Goddard Space Flight Center \\ Greenbelt, MD 20771
}

\begin{abstract}
The Laser Interferometer Space Antenna mission is a planned gravitational wave detector consisting of three spacecraft in heliocentric orbit. Laser interferometry is used to measure distance fluctuations between test masses aboard each spacecraft to the picometer level over a 5 million kilometer separation. Laser frequency fluctuations must be suppressed in order to meet the measurement requirements. Arm-locking, a technique that uses the constellation of spacecraft as a frequency reference, is a proposed method for stabilizing the laser frequency. We consider the problem of arm-locking using classical optimal control theory and find that our designs satisfy the LISA requirements.
\end{abstract}

\section{INTRODUCTION}

The Laser Interferometer Space Antenna (LISA) is a space-based gravitational wave detector under joint development by NASA and ESA [1-3]. The goal is to measure gravitational waves, ripples in the curvature of space-time produced by astrophysical events such as black hole collisions. Gravitational waves produce an oscillating tidal strain in space, the effect of which is to modulate the distance between a set of separated inertial masses. The amplitude of the oscillations is proportional to the nominal distance between the masses. Therefore the wave's amplitudes are characterized by the strain, or ratio of change in length to nominal length. For astrophysical sources of interest to LISA, typical strain amplitudes are $10^{-21}$ and have Fourier frequencies between $10^{-4} \mathrm{~Hz}$ and $10^{-1} \mathrm{~Hz}$.

The LISA instrument consists of a triangular constellation of three spacecraft in heliocentric orbit at $1 \mathrm{AU}$, trailing approximately 20 degrees behind Earth. Each arm in the constellation is nominally 5 million km long and varies by as much as one percent with an annual period. Laser interferometry is used to monitor distance fluctuations between freely floating masses that are contained within the spacecraft at each end of the arm and are isolated from external disturbances using the technique of drag-free control [4,5]. A measurement resolution of approximately $10 \mathrm{pm}(1 \mathrm{pm}=$ $10^{-12} \mathrm{~m}$ ) is required for the interferometric measurement.

LISA interferometry utilizes coherent optical transponders in order to compensate for the large diffraction losses caused by the immense distances between the spacecraft. A schematic representation of the LISA interferometer (restricted to two arms) is shown in Figure 1. Each of the three spacecraft, labeled ' $A$ ',' $B$ ',' $C$ ', contain two instrumental payloads labeled 'A1', 'A2', 'B1', etc. These payloads consist of laser, an optical bench, and a phase measurement system. The phase-measurement system measures the phase difference between the local laser and the laser on the adjacent bench as well as the phase difference between the local laser and the received beam from the far spacecraft. These measurements are manipulated and combined to generate signals that suppress laser phase noise while retaining the gravitational wave signals.

For example, consider the laser associated with payload A1 on spacecraft A as the 'master' laser. The back-link fiber measurement is used to measure the phase difference between lasers A2 and A1. By adjusting the phase of laser A2 to track that of laser A1 (a phase-locked loop), the two beams traveling to the distant spacecraft behave as if they originated from the same laser. Similarly, lasers B1 and C1 track the incoming beam from the spacecraft A. With all of these loops in operation, the two remaining phase measurements on spacecraft A produce the following signals:

$$
\phi_{A j}(t)=\phi(t)-\phi\left(t-\tau_{A j}\right) ; j=B, C
$$

Where $\phi(t)$ is the phase of the master laser and $\tau_{A j}$ is the round-trip light travel time between spacecraft $\mathrm{A}$ and $\mathrm{j}$. 
Each of these signals contains the desired strain information but is dominated by the random fluctuations of the laser phase. A technique known as Time Delay Interferometry (TDI) $[6,7]$ combines the two signals with varying time delays in order to suppress these fluctuations while retaining the gravitational wave signal. The noise suppression of TDI is primarily limited by knowledge of the nominal arm lengths, $\tau_{A j}$. With the assumption that a ranging system can track these arm lengths to $1 \mathrm{~m}$, TDI can meet the LISA measurement requirements if the phase fluctuations of the master laser are below

$$
\frac{282 \mathrm{~Hz}}{\sqrt{H z}} \sqrt{\left(1+\left(\frac{2 \mathrm{mHz}}{\mathrm{f}}\right)^{4}\right)} ; 0.1 \mathrm{mHz} \leq f \leq 100 \mathrm{mHz}
$$

The expected fre-running noise of the LISA lasers is $10 \mathrm{kHz} / \sqrt{\mathrm{Hz}}\left(\frac{1 \mathrm{~Hz}}{\mathrm{f}}\right)$, hence it is necessary to actively stabilize the laser frequency in order to meet the above requirement. Several methods of frequency stabilization have been proposed, including traditional laboratory techniques such as spectroscopic references [8] and high-finesse optical cavities [9]. Here we consider a technique known as arm-locking [10-17], which uses the arms of the LISA constellation as a frequency reference.

The basic concept of arm-locking is to use the information contained in the phasemeter signals $\phi_{A j}$ to estimate the phase/frequency noise of the master laser and thereby correct it. This concept was first proposed by Sheard, et al. [10] and has subsequently been further refined and studied by others [11-17]. In this work, we apply the techniques of classical optimal control theory to this problem.

\section{ARM LOCKING}

For this initial study, the phase-locked loops for the local and remote lasers are not modeled. This assumption should not impact the developments presented herein as their contribution is well above the LISA measurement band. Additional noise sources that enter the phasemeter measurements on spacecraft $A$ are considered, leading to a refinement of the laser phase dynamics.

- $\quad v_{\text {laser }}$ denotes the uncompensated frequency noise of the master laser.

- $\quad v_{S C}^{A B}$ represents the frequency noise induced by the motion of the spacecraft $A$ with respect to spacecraft $B$ along the $A B$ arm.

- $\quad v_{S C}^{A C}$ represents the frequency noise induced by the motion of the spacecraft $\mathrm{A}$ with respect to spacecraft $\mathrm{C}$ along the $A C$ arm.

- $\quad v_{c l o c k}^{A B}$ denotes the frequency noise brought on due to the uncertainty in the clock in the AB phasemeter readout.

- $\quad v_{\text {clock }}^{A C}$ denotes the frequency noise brought on due to the uncertainty in the clock in the AC phasemeter readout.

- $v_{\text {shot }}^{A B}$ represents the shot noise in the AB phasemeter readout.

- $v_{\text {shot }}^{A C}$ represents the shot noise in the AC phasemeter readout.

The parameter "ALS" refers to the arm locking sensor, which is designed to recover the optical phase within the LISA bandwidth using the outputs of the phasemeters. The parameter " $\mathrm{K}(\mathrm{s})$ " denotes the compensator for the arm locking loop. The internal dynamics of the phasemeter, the actuation system for laser frequency control, and the phase-locked loops at the local and remote spacecraft, are not considered in the paper, as they do not have any significant impact to the 
design or performance of the ALS and the compensator in the LISA band. Here, the dynamics of the optical signals is expressed in terms of frequency for convenience. The outputs of the phasemeters are assumed to be processed to act as a frequency-meter. The outputs of the phasemeters are given as

$$
\begin{aligned}
& P_{A B}(s)=\left(1-e^{-s \tau_{A B}}\right) v_{1}(s)+v_{\text {clock }}^{A B}(s)+v_{\text {shot }}^{A B}(s)+v_{S C}^{A B}(s) \\
& P_{A C}(s)=\left(1-e^{-s \tau_{A C}}\right) v_{1}(s)+v_{\text {clock }}^{A C}(s)+v_{\text {shot }}^{A C}(s)+v_{S C}^{A C}(s)
\end{aligned}
$$

The ALS output blends the signals from the phasemeters, i.e.,

$$
S(s)=\left[S_{A B}(s) \quad S_{A C}(s)\right]\left\{\begin{array}{l}
P_{A B}(s) \\
P_{A C}(s)
\end{array}\right\}
$$

Where $S_{A B}(s)$ and $S_{A C}(s)$ represent the elements of the ALS corresponding to the AB and AC phasemeters, respectively. The closed-loop system dynamics may now be written as

$$
[1+K(s) G(s)] v_{1}(s)=-K(s) S_{A B}(s) v_{n o i s e}^{A B}(s)-K(s) S_{A C}(s) v_{n o i s e}^{A C}(s)+v_{l a s e r}(s)
$$

Where

$$
\begin{aligned}
G(s)= & S_{A B}(s) L_{A B}(s)+S_{A C}(s) L_{A C}(s) \\
& L_{A B}(s)=\left(1-e^{-s \tau_{A B}}\right) \\
& L_{A C}(s)=\left(1-e^{-s \tau_{A C}}\right) \\
v_{\text {noise }}^{A B}(s)= & v_{\text {clock }}^{A B}(s)+v_{\text {shot }}^{A B}(s)+v_{S C}^{A B}(s) \\
v_{\text {noise }}^{A C}(s)= & v_{\text {clock }}^{A C}(s)+v_{\text {shot }}^{A C}(s)+v_{S C}^{A C}(s)
\end{aligned}
$$

The term $[1+K(s) G(s)]$ is the return difference, and $K(s) G(s)$ denotes the loop gain for the arm locking loop. The laser noise with arm locking loop closed is written as

$$
\begin{gathered}
v_{1}(s)=-[1+K(s) G(s)]^{-1} K(s)\left[S_{A B}(s) v_{\text {noise }}^{A B}(s)+S_{A C}(s) v_{\text {noise }}^{A C}(s)\right] \\
+[1+K(s) G(s)]^{-1} v_{\text {laser }}(s)
\end{gathered}
$$

The chal lenge is to design an ALS, S(s), and a compensator, $K(s)$, which would stabilize $v_{1}(s)$ to below required levels in the LISA band. 


\section{NOISE MODELS}

\section{Laser Noise}

Arm locking can be implemented for a free running laser, or a laser that is pre-stabilized to a local reference, e.g., cavity. Although the latter option provides a more benign noise environment for arm locking, it adds additional complexities, such as hardware, mass, cost, etc. Moreover, since one of the objectives of the paper is to assess the feasibility of arm locking to meet LISA's laser noise stability requirements, the laser is assumed to be free running, with no prestabilization. This represents a more challenging case for arm locking. The laser frequency noise model is based on laboratory measurements of a free running Nd:YAG NPRO laser. The spectrum of the noise drops with a slope of 1/f, and is provided below.

$$
v_{\text {laser }}=10 \mathrm{kHz} / \sqrt{\mathrm{Hz}}\left(\frac{1 \mathrm{~Hz}}{f}\right)
$$

\section{Shot Noise}

Shot noise enters as additive noise at each phasemeter measurement. It is due to the quantization of detected photons in the photoreciever and affects both the amplitude and phase of the signal. For an interference of two beams with powers $P_{1}$ and $P_{2}$, assuming perfect contrast, the shot noiæe will affect the phase as

$$
\phi_{\text {shot }}=\sqrt{\frac{h c\left(\frac{1}{P_{1}}+\frac{1}{P_{2}}\right)}{\eta \lambda}}
$$

Where $h$ is Planck's constant, $c$ is the speed of light, $\eta$ is the quantum efficiency of the detector, and $\lambda$ is the wavelength of the light. Putting in the constants for our 1 micron light and assuming $\eta=1$ the level is

$$
\phi_{\text {shot }}=69 n \text { Cycles } / \sqrt{H z} \sqrt{1 \mu W\left(\frac{1}{P_{1}}+\frac{1}{P_{2}}\right)}
$$

This noise will be white in Fourier frequencies up to optical frequencies where it will roll-off. The statistics will be somewhat complicated but we can assume that we have enough photons that a Gaussian approximation is sufficient. To convert this to a frequency noise in $\mathrm{Hz}$, we simply multiply by $2^{*} p i^{*} f$,

$$
v_{\text {shot }}=0.43 \mu \mathrm{Hz} / \sqrt{\mathrm{Hz}}\left(\frac{f}{1 \mathrm{~Hz}}\right) \sqrt{1 \mu W\left(\frac{1}{P_{1}}+\frac{1}{P_{2}}\right)}
$$

For LISA, we can assume $100 \mathrm{pW}$ of light received from the far SC is interfered with a local beam of $\sim 1 \mathrm{~mW}$. This gives a frequency shot noise of $43 \mu \mathrm{Hz} / \mathrm{rtHz}^{*}(\mathrm{f} / 1 \mathrm{~Hz})$. 


\section{Clock Noise}

Clock noise enters in the phasemeter. This can affect both the PLLs at the far SC and the arm-locking sensor. A rough estimate of the magnitude of the clock noise is

$$
v_{\text {clock }}=3 \mu \mathrm{Hz} / \sqrt{\mathrm{Hz}}\left(\frac{v_{\text {beat }}}{10 \mathrm{MHz}}\right) \sqrt{\frac{1 \mathrm{~Hz}}{f}}
$$

Where $v_{\text {beat }}$ is the frequency of the beat note. Here, a range of 5-15 MHz is used for $v_{\text {beat }}$. The clock noise will be independent on each spacecraft. For arm-locking it should be correlated between the two phasemeter signals at the corner spacecraft.

\section{Spacecraft Motion}

The current LISA baseline calls for using the bench-to-bench measurement to form the arm-locking error signals. This means that the spacecraft motion will be present in the sensors. This noise should be added as a varying time-delay between the spacecraft. In other words, it affects the transfer function of the arm rather than the noise. However, given its small size ( $2.5 \mathrm{~nm} / \mathrm{rtHz})$ it is reasonable to treat it as an additive noise term in the phasemeter signal. The noise can be approximated by the Doppler effects of spacecraft motion as perceived in the phasemeter.

$$
v_{S C}=\left(\frac{2 \pi}{\lambda}\right) 2.5 \times 10^{-9} \mathrm{~Hz} / \sqrt{H z}\left(\frac{f}{1 H z}\right)
$$

With $\lambda=1.064 \mu m$,

$$
v_{s C}=14.8 \mathrm{mHz} / \sqrt{H z}\left(\frac{f}{1 H z}\right)
$$

\section{ARM LOCKING SENSOR}

The arm locking sensor (ALS) is a filter or software which uses the measurement data from the $A B$ and $A C$ phasemeters to provide an estimate of the current laser frequency/phase, with some acceptable latency. This estimate is used by the dual arm locking compensator to correct for frequency/phase errors. The approach taken here for the design of ALS is based on optimal estimation theory. Assuming a discrete-time implementation of the estimation scheme, let $\Delta t$ denote the sampling time. Then, the phasemerter dynamics provided in Eqs. (2) and (3) may be approximated in the z-domain by

$$
\begin{aligned}
& P_{A B}(z)=\left(1-z^{-k_{A B}}\right) v_{1}(z)+v_{n o i s e}^{A B}(z) \\
& P_{A C}(z)=\left(1-z^{-k_{A C}}\right) v_{1}(z)+v_{\text {noise }}^{A C}(z)
\end{aligned}
$$


Where $k_{A B}=\operatorname{ceil}\left(\tau_{A B} / \Delta t\right)$ and $k_{A C}=\operatorname{ceil}\left(\tau_{A C} / \Delta t\right)$. Without any loss in generality, assume $k_{A B}>k_{A C}$. Note that the discrete representations in Eqs. (7) and (8) should not impact laser frequency/phase estimation in the critical frequency band, which lies at or around the LISA band, if $\Delta t$ is chosen small enough such that $1 / \Delta t$ is sufficiently outside the band of interest. With the phasemeter dynamics defined in the above, the dynamics of the arm locking loop may be written in state-space form

$$
\begin{gathered}
x_{k+1}=A x_{k}+B u_{k}+\Gamma \mathrm{w}_{\mathrm{k}} \\
y_{k}=C x_{k}+D u_{k}+H \mathrm{w}_{\mathrm{k}}+\mathrm{v}_{\mathrm{k}}
\end{gathered}
$$

Where the state and measurement vectors are defined as

$$
\begin{aligned}
& x_{k}=\left\{\begin{array}{llll}
v_{1 k} & v_{1 k-1} & \ldots & v_{1_{k-k_{A B}}}
\end{array}\right\}^{T} \\
& y_{k}=\left\{\begin{array}{ll}
P_{A B_{k}} & P_{A C_{k}}
\end{array}\right\}^{T}
\end{aligned}
$$

The state vector includes the laser frequency at the current cycle, the laser frequency from the previous cycle, and so on, all the way to the laser frequency from $k_{A B}$ cycles ago. However, note that the current cycle is delayed from the current time by one cycle, as it does not include the instantaneous impact of laser frequency actuation and laser noise. The state matrix $A$ is sparse with non-zero elements below the diagonal, i.e.,

$$
\begin{aligned}
& A=\left[\begin{array}{ccccc}
0 & 0 & \cdots & \cdots & 0 \\
1 & 0 & \cdots & \cdots & 0 \\
0 & 1 & \ddots & \cdots & \vdots \\
\vdots & 0 & \ddots & \cdots & \vdots \\
\vdots & \vdots & \ddots & 0 & \vdots \\
0 & 0 & \cdots & 1 & 0
\end{array}\right] \\
& B=\left[\begin{array}{c}
1 \\
0 \\
\vdots \\
0
\end{array}\right] ; \quad \Gamma=\left[\begin{array}{c}
1 \\
0 \\
\vdots \\
0
\end{array}\right] \\
& C=\left[\begin{array}{lllllll}
0 & 0 & \cdots & \cdots & \cdots & 1 \\
0 & 0 & \cdots & 1 & \cdots & 0
\end{array}\right] \quad ; \quad D=\left[\begin{array}{l}
1 \\
1
\end{array}\right] ; \quad H=\left[\begin{array}{l}
1 \\
1
\end{array}\right]
\end{aligned}
$$

Note the non-zero element in the first row of the output influence matrix, $\mathrm{C}$, is the last element, corresponding to the state at $k_{A B}$ cycles ago, while the non-zero element in the second row is element no. $k_{A C}$. Finally, the process noise vector, $\mathbf{w}_{\mathbf{k}}$, and the measurement noise vector, $\mathrm{v}_{\mathbf{k}}$, are defined as

$$
\begin{gathered}
w_{k}=v_{\text {laser }_{k}} \\
v_{k}=\left\{\begin{array}{ll}
v_{\text {noise }_{k}}^{A B} & v_{\text {noise }_{k}}^{A C}
\end{array}\right\}^{T}
\end{gathered}
$$


The ALS must provide an estimate of the laser frequency to be used in the arm locking controls loop. To accomplish this, optimal filtering is proposed. In particular, since only steady-state processes are involved and/or are of main concern, Steady-state Kalman filtering is used to compute an estimate of the laser frequency [18-19]. The steady-state Kalman filter may be realized in state-space form and is represented by the following equations.

$$
\begin{gathered}
\hat{x}_{k+1}(-)=A \hat{x}_{k}(-)+B u_{k}+\mathrm{L}\left(y_{k}-\mathrm{C} \hat{x}_{k}(-)-\mathrm{D} u_{k}\right) \\
\hat{x}_{k}(+)=\hat{x}_{k}(-)+\mathrm{M}\left(y_{k}-\mathrm{C} \hat{x}_{k}(-)-\mathrm{D} u_{k}\right) \\
\hat{y}_{k}=C \hat{x}_{k}(+)+D u_{k}
\end{gathered}
$$

Here the vector $\hat{x}_{k}(-)$ represents an optimal estimate of the state vector $x_{k}$ based on all prior measurements, while the vector $\hat{x}_{k}(+)$ represents an optimal estimate which includes the current measurement as well. Equation (11) provides the time update, while Eqs. (12) and (13) provide the measurement updates. The only estimate that is of concern for ALS is the most current estimate of the laser frequency the Kalman filter can provide. And that estimate is the first element of the vector $\hat{x}_{k}(+)$.

\section{Filter Realization}

It can be shown that due to the sparse structure of the plant, represented by Eq. (9) and (10), the implementation of the optimal ALS (OALS) filter may be separated from the control input, such that the OALS filter assumes the following from.

$$
\begin{gathered}
\hat{x}_{k+1}(-)=A \hat{x}_{k}(-)+\mathrm{L}\left(y_{k}-\mathrm{C} \hat{x}_{k}(-)\right) \\
v_{1_{k}}(+)=v_{1_{k}}(-)+\mathrm{M}_{1}\left(y_{k}-\mathrm{C} \hat{x}_{k}(-)\right)
\end{gathered}
$$

In other words, one can show that for every controller and filter combination for the system in Eqs. (11)-(13) there is an equivalent filter and controller combination, with a filter represented by Eqs. (14) and (15). Of course, one can continue to use the filter equations (11)-(13), which includes the control signal, or a combination of the two implementation options.

The resulting OALS filter, represented by Eqs. (11)-(13), or Eqs. (14)-(15), can be high order, depending on the sample time $\Delta t$. However, the system matrices of the ALS filter are sparse, and hence the al gebraic propagation can be done quite efficiently for flight software implementation using sparse matrix operations. Moreover, the dynamic range of interest resides primarily at or around the LISA measurement band. As such, a balanced reduction procedure may be followed to obtain a low-order approximation of the OALS filter for implementation and analysis. Reference [20] provides a thorough description of bal anced realization and reduction process. 


\section{COMPENSATOR DESIGN}

The arm locking controller uses the estimated frequency error provided by the OALS, save any commanded offset, to adjust the frequency of the laser. The laser actuation dynamics, which may include thermal and piezo actuations, is not included in the compensator design or follow-up analysis. The objective of the compensator design is to suppress the laser frequency noise to a level below pre-TDI requirement (Eq. (1)), while ensuring that the sensing-related noise, e.g., clock noise, shot noise, spacecraft motion noise, does not amplify unreasonably outside the bandwidth of interest. Furthermore, the arm locking compensator has to contend with and suppress spikes in the plant dynamics, within the LISA band, that are brought on by the zeros due to round-trip delays in the two arms. The compensator has to provide at least $6 \mathrm{~dB}$ of gain margin and $30^{\circ}$ of phase margin for the arm locking loop. The arm locking compensators were designed using the classical frequency domain approach. The designed compensators generally include a low-frequency lead-lag/lag component, followed by a first-order attenuation filter.

\section{ANALYSIS RESULTS AND DISCUSSION}

The proposed arm locking control strategy was applied to a LISA constellation configuration. Here, the round-trip times for the laser signals were assumed to be nominally at 33 and 32.4 seconds, i.e., $\tau_{A B}=33$ and $\tau_{A C}=32.4$. The optimal arm locking sensor was designed using the optimal estimation approach described earlier. The sample time for filter was chosen at $0.1 \mathrm{sec}$, leading to a filter order of 331 . The noise models used for the ALS design were similar to those described earlier. The models were an exact match within the LISA band. However, below and above band the emphasis on some models were changed to provide desired loop shaping. The filter implementation was based on a partial control signal feedback (see Eqs. (11)-(13)). A compensator was designed, whose Bode plot is shown in Fig. 2. As expected, the highest gain is concentrated bel ow and within the LISA band. This OALS filter and compensator combination is referred to as controller $\mathrm{K} 1$. Figure 3 shows the loop gain, with the attained stability margins. Both gain and phase margins meet the requirements. Laser frequency noise suppression is demonstrated in Fig. 4. It is observed that the laser frequency noise is well below $282 \mathrm{~Hz} / \sqrt{\mathrm{Hz}}$ throughout the LISA band, and hence meets LISA's pre-TDI requirements. The response is dominated mostly by the free-running laser frequency noise. The contributions from the clock noise and spacecraft jitter provide a floor for noise suppression. Note that the sequence of peaks within and close to the LISA band occur at the frequencies equal to multiples of the inverse round-trip delay of the arms. These peaks have been effectively suppressed by using the estimated laser frequency from OALS. This is made possible by using phase/frequency information from two arms. If one had to resort to single arm locking, then these peaks are not amenable to suppression as they lie on the zeros of the plant dynamics at multiples of the inverse round-trip delay.

\section{ALS Sensitivity}

The arm locking sensor filter is based on discrete-time steps, which means that round-trip time for each arm, and ther fore the difference between them, is captured at that quantization level. Since practical considerations, such as flight software implementation, limit the fineness of the step-size, it is imperative to assure the OALS is robust to errors in the representation of the round-trip times, and hence the difference between them.

The closed-loop arm locking anal ysis was repeated with controller $\mathrm{K} 1$, which was designed based on $\tau_{A B}=33$ and $\tau_{A C}=32.4$. However, for the analysis $\tau_{A C}$ was set to 32.5 , resulting in the difference in round-trip times of 0.5 seconds. The performance of arm locking controller is provided in Fig. 5, where little or no change is observed in the level of laser frequency suppression from the nominal case. Hence, the design appears to be insensitive to representation errors in the round-trip time for a single arm, or the difference in the round-trip times between the two arms, of $0.1 \mathrm{sec}$ or smaller. Onorbit implementation would switch the OALS filter and compensators for their most optimal at a time quantization of equal or better than 0.1 seconds. Therefore, the proposed OALS filter and compensation approach is feasible for on-orbit implementation.

In order to exercise the range of feasibility of the OALS filter, a new analysis was performed with controller K1. However, this time $\tau_{A C}$ was set to 32.95, resulting in the difference in round-trip times of 0.05 seconds. Figure 6 
illustrates the laser noise frequency suppression for this setup. Although LISA pre-TDI requirements are met for most of the LISA band, however significant poke-throughs are observed at or near the multiples of inverse round-trip times. Next, a new OALS filter and compensator were designed using the best representation of the round-trip times for the arms. This representation set the values of $\tau_{A B}$ and $\tau_{A C}$ to $33 \mathrm{sec}$. and $32.9 \mathrm{sec}$., respectively. The performance of the optimal filter is shown in Fig. 7. It is observed that the laser frequency noise is suppressed well below $282 \mathrm{~Hz} / \sqrt{\mathrm{Hz}}$ throughout the LISA band, and hence meets LISA's pre-TDI requirements.

As the differential arm length approaches zero, the ripple in the closed-loop OALS system near the $1 / \tau$ frequencies increases and eventually becomes larger than the free-running noise. This is because the nulls in the individual arm responses are degenerate and no combination of the two can be used to measure phase noise at these Fourier frequencies. This is a common feature of all loops that utilize two LISA arms [15]. In the nominal case when all three LISA arms are available, it will be possible to select the master SC to ensure that the differential arm-length is sufficiently great to avoid this problem. However, in the event of a loss of one of the arms, the master SC is fixed and there will be times when the differential arm-length goes to zero.

By systematically varying the differential arm-length, it was found that the OALS filter presented above will meet the pre-TDI frequency noise requirements so long as the differential arm-length exceeds $2 \mathrm{msec}$. The fraction of the orbit with differential arm-lengths smaller than this varies depending on the arm that is lost and the exact orbital model. Using a simplified model (first-order in eccentricity, no perturbations from outer planets, etc.), it is estimated that a differential arm-length of less than $2 \mathrm{msec}$ will last for a period of $\sim 1 / 2$ day. Therefore, in the case that a link is lost, the OALS filter will still satisfy mission requirements for $99.9 \%$ of the mission duration.

\section{CONCLUDING REMARKS}

The problem of arm-locking for LISA using phasemeter/frequency-meter data from two of its arms was considered. Using classical optimal estimation theory, an approach for designing optimal filters to recover the laser frequency noise within the LISA band was presented. Feasible compensators for the arm locking loop were designed and implemented. . Numerical analysis demonstrated that the proposed strategy to use optimal estimation of laser phase/frequency for arm locking applications is feasible. It is robust to variations in representation errors for round-trip times of the arms, and has the advantage of not requiring precise knowledge of the round-trip times or their difference.

An important effect that has recently been recognized for arm-locking is that of Doppler estimation error in the phasemeter. Variations in the relative velocity betwen the spacecraft add a time-varying Doppler shift to the phasemeter signal. This component is subtracted from the phasemeter signal using an orbital model. This model is not perfect however and some residual Doppler shifts remain and affect both the residual noise and dynamic range of the laser. This effect will be considered in subsequent studies.

\section{REFERENCES}

1. J. Faller, P. Bender, J. Hall, D. Hils, M. Vincent, Space antenna for gravitational wave astronomy, in: Colloquium on Kilometric Optical Arrays in Space, ESA-SP 226 (1985).

2. P. Bender, K. Danzmann, and the LISA Study Team, "Laser Interferometer Space Antenna for the Detection and Observation of Graviational Waves, pre-Phase A report," Tech. Rep. MPQ233, Max-Planck-Institute for Quantum Optics, Garching (1998). 2nd ed.

3. Shaddock, D.; "Space based gravitational wave detection with LISA", Class. Quantum Grav. 25114012 (11pp) 2008.

4. Lange, B.; "The drag-free satellite"; AIAA J. 2 1590-606, 1964.

5. T. Hyde, P. Maghami and S. Merkowitz; "Pointing Acquisition and Performance for the Laser Interferometer space antenna mission", Class. Quantum Grav., 21 S635-S640, 2004.

6. Tinto, M., Estabrook, F., and Armstrong J.; 2004, Time delay interferometry with moving spacecraft arrays Phys. Rev. D 69082001.

7. Shaddock, D., Ware, B., Spero, R., and Vallisneri, M.; 2004, Postprocessed time-delay interferometry for LISA Phys. Rev. D 70081101. 
8. Leonhart, V., and Camp, J.; "Space interferometry application of laser frequency stabilization with molecular iodine"; Appl. Opt. 45, 4142-4146 (2006).

9. McNamara, P., Ward, H., Hough, J., Robertson, D.; "Laser frequency stabilization for spaceborne gravitational wave detectors", Class. Quantum Grav. 14, 1543-1547 (1997).

10. Sheard, B., Gray, M., McClelland, D., and Shaddock, D.; 2003 Laser frequency stabilization by locking to a LISA arm Phys. Lett. A 320 9-21.

11. Sylvestre, J.; 2004, Simulations of laser locking to a LISA arm Phys. Rev. D 70102002.

12. Mueller G, McNamara P, Thorpe Jl, and Camp J 2005 "Laser Frequency Stabilization for LISA" NASA/TM-2005-212794.

13. Marin, A. et al; 2005, Phase locking to a LISA arm: first results on a hardware model Class. Quantum Grav. 22 S235-42.

14. M. Herz; Opt. Eng. (Bellingham, Wash.) 44, 090505 (2005).

15. Sheard, B., Gray, M., and McClelland D.; 2006, High-bandwidth laser frequency stabilization to a fiber-optic delay line Appl. Opt. $458491-9$.

16. Thorpe, J., and Mueller, G., 2005; Experimental verifcation of arm-locking for LISA using electronic phase delay Phys. Lett. A 342 199-204.

17. Sutton, A., Shaddock, D.; Laser frequency stabilization by dual arm locking for LISA; Phys. Rev. D 78, 082001 (2008).

18. Stengel, R. F.; "Stochastic Optimal Control: Theory and Application", John Wiley and Sons, Inc., NY, 1986.

19. Phillips, C. L., and Nagle, H. T.; "Digital System Analysis and Design", Third Edition, Prentice Hall, Englewood Cliffs, NJ, 1995.

20. Maciejowski, J. M.; "Multivariable Feedback design", Addison-Wesley, 1989.

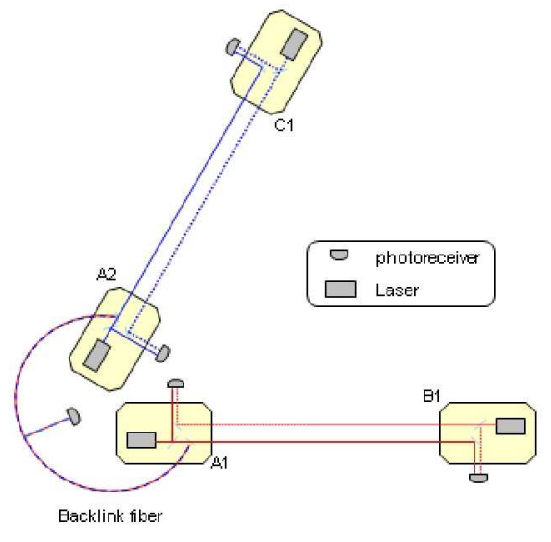

Figure 1 Block Diagram for Arm Locking

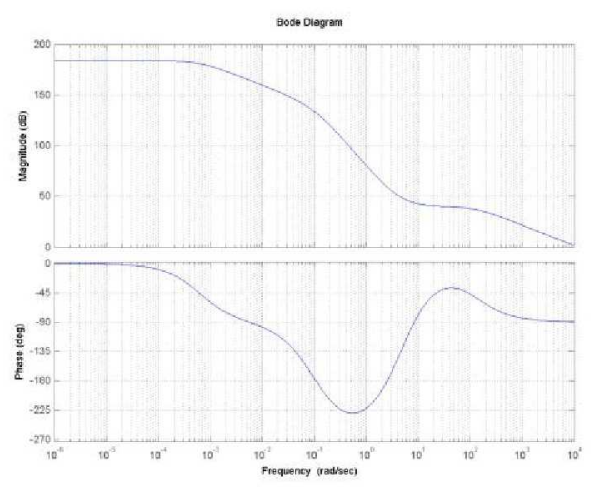

Figure 2 Bode Plot for the Arm Locking Compensator 


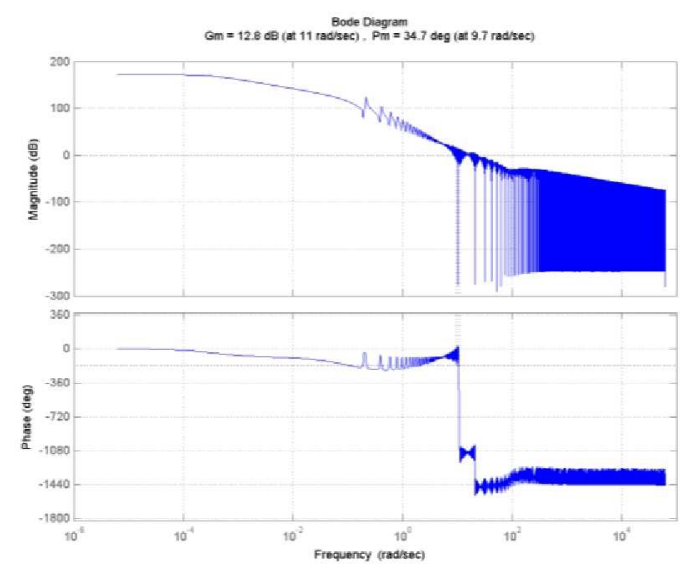

Figure 3 Loop Gain for the Arm Locking Loop

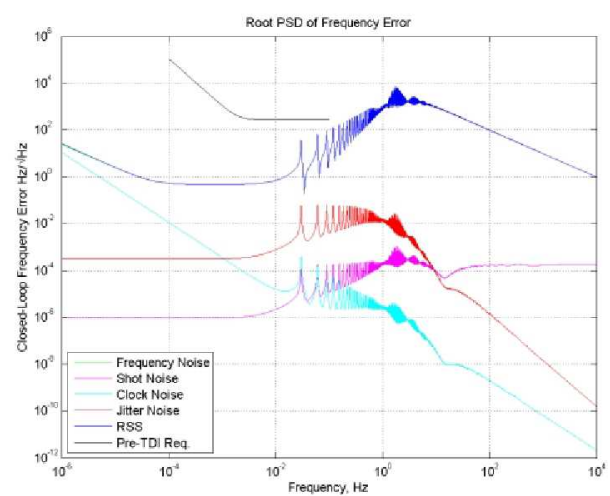

Figure 5 PSD for Laser Noise Frequency: Controller K1,

$\tau_{A B}=33$ and $\tau_{A C}=32.5$

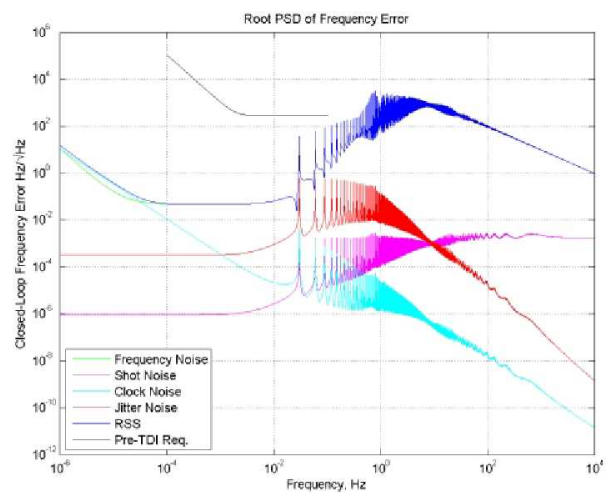

Figure 7 PSD for Laser Noise Frequency: Optimal Controller,

$\tau_{A B}=33$ and $\tau_{A C}=32.95$

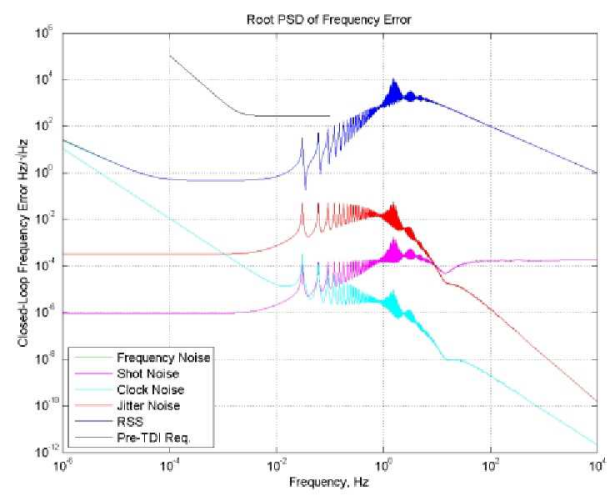

Figure 4 PSD for Laser Noise Frequency: Controller K1

$\tau_{A B}=33$ and $\tau_{A C}=32.4$

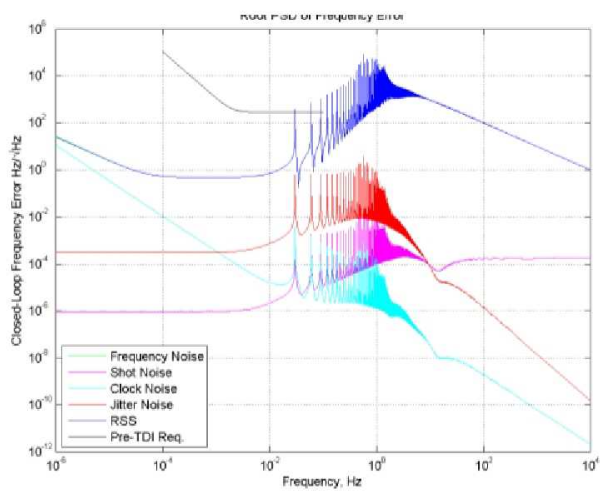

Figure 6 PSD for Laser Noise Frequency: Controller K1,

$\tau_{A B}=33$ and $\tau_{A C}=32.95$ 